\title{
„ARME“ UND „REICHE“ GRÄBERFELDER DER WIELBARK-KULTUR - EINIGE BEISPIELE
}

\author{
M A G D A L E N A M A C Z Y ŃS K A
}

\begin{abstract}
'Poor' and 'Rich' Burial Grounds of the Wielbark Culture - a Few Examples. Cemeteries attributed to the Wielbark culture - similarly to all other cemeteries - could be divided into those well- and poorly equipped. Interestingly, however, while in some cemeteries there are at least a couple of lavishly equipped graves, in others - even located in the close neighbourhood - poorly equipped graves dominate. This phenomenon has been observed in several cases and is difficult to explain with the current state of research. It seems that the differences in equipment at various cemeteries reflect the social stratification and the fact that groups of various economic statuses cohabited in the near vicinity.
\end{abstract}

Keywords: Poland, Roman Period, Wielbark culture, burial grounds, burial customs.

Als ich vor vielen Jahren, nach einigen Grabungskampagnen auf dem Gräberfeld in Babi Dół-Borcz in Pommern und von der Armut der dortigen, oft auch beigaben losen Bestattungen etwas deprimiert, Ryszard Wołągiewicz, den Ausgräber u. a. einer anderen „armen” Nekropole - von Grzybnica fragte, welche Ursachen hinter den arm und reich ausgestatteten Gräbern stehen können, habe ich die folgende Antwort erhalten: „Es ist noch zu früh, darüber nachzudenken." Diese Worte wurden den Anlass, diese kleine Studie zu schreiben.

Im ganzen barbarischen Europa der römischen Kaiserzeit sind Gräberfelder mit ärmerer und reicherer Ausstattung, aber auch Einzelbestattungen der gesellschaftlichen Eliten bekannt. Die Studien zur Sozialstruktur erfreuen sich einer umfangreichen Literatur, von der nur die neueren und die römische Kaiserzeit betreffenden Studien angeführt seien (Becker 2005; von Carnap-Bornheim 2006; Gebühr 1976; Hedeager 1980; Kunst 1978; Nagy 2007; Quast 2009; Schuster 2010; Skóra 2015; Steuer 1982).

Heute ist der Forschungsstand zu den Nekropolen der Wielbark Kultur im Vergleich zu den 60-er und 70-er Jahren, als die nachkriegszeitlichen Ausgrabungen auf einigen Gräberfeldern begonnen haben, unvergleichbar besser und wir können versuchen, Überlegungen zu folgendem Problem anzustellen: Worin liegt der Grund, dass benachbarte, manchmal nur einige Kilometer voneinander entfernte Nekropolen mit etwa gleicher Belegungsdauer so verschieden ausgestattet sind? Es gibt Gräberfelder mit reichen bzw. sehr reichen Beigaben, während die benachbarten eine arme oder eine einfach durchschnittliche Ausstattung aufweisen.
Gleich jetzt müssen wir festhalten, dass an dieser Stelle statistischen Abrechnungen betreffs der Quantität reicher, durchschnittlich ausgestatteter und armer Gräber auf einem Gräberfeld nicht verwenden werden können, da bisher nur zwei Gräberfelder mit Hügeln und Steinkreisen vollständig bzw. fast vollständig ausgegraben worden sind: Babi Dół-Borcz, Kr. Kartuzy, zur Publikation von mir und I. Jakubczyk bereits vorbereitet und Nowy Łowicz, Kr. Drawsko Pomorskie, von A. Cieśliński und A. Kasprzak bearbeitet (s. unten). Von den flachen Nekropolen sind Kowalewko, Kr. Oborniki (Skorupka 2001) und Niedanowo, Kr. Nidzica (Ziemlińska-Odojowa 1999) vollständig untersucht worden. Es folgen fast gänzlich ausgegrabene Gräberfelder, wie Cecele, Kr. Siemiatycze (Jaskanis 1996) und Nadkole, Kr. Węgrów (Andrzejowski/Żórawska 2002). Die systematisch untersuchten Nekropolen, wie z. B. Lubowidz und Pruszcz Gdański, Fpl. 10 (s. unten) wurden vor Grabungsbeginn teilweise zerstört, während andere im heute bebauten Areal liegen (Abb. 1).

Als erstes Beispiel werden hier zwei Nekropolen an der Ostseeküste, Czarnówko und Lubowidz, $\mathrm{Kr}$. Lębork, angeführt, die $4 \mathrm{~km}$ voneinenander entfernt liegen. Czarnówko, das bisher größte Gräberfeld der Wielbark-Kultur, zählt mindestens 2400 Bestattungen (Schuster 2014; 2018). Neun davon waren ausserordentlich reich ausgestattet und bargen Silber-, Bronze- und Glasgefässe, darunter solche Unikate wie einen Bronzekessel mit suebischen Köpfen, ein Glasgefäss in Fischform und einen Glasbecher mit Schwanendarstellungen. Es folgen goldene und silberne Trachtbestandteile, die alle in die fortgeschrittene Stufe B 2 bzw. in die 


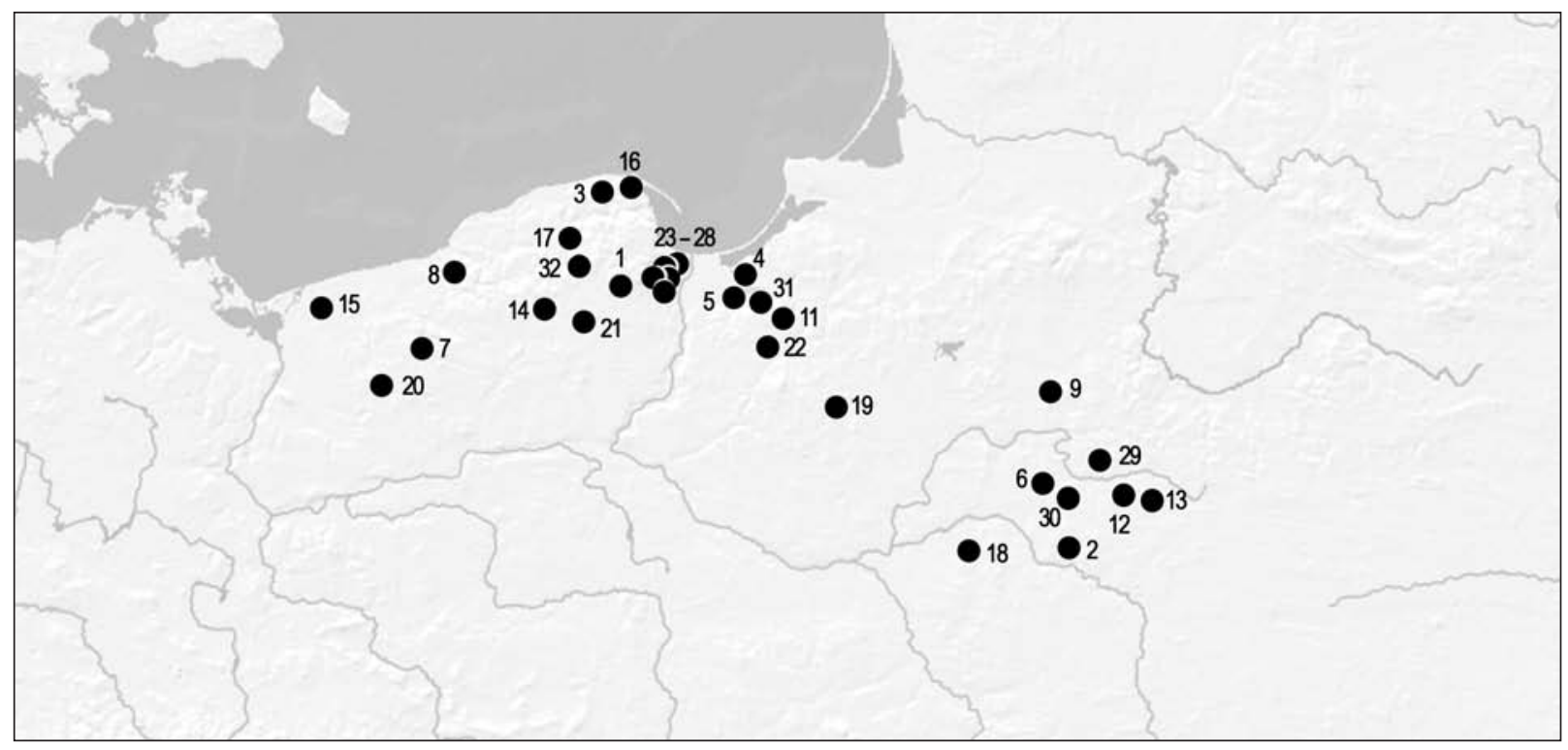

Abb. 1. Im Text erwähnte Gräberfelder der Wielbark-Kultur. 1 - Babi Dół-Borcz; 2 - Cecele; 3 - Czarnówko; 4 - Elbląg-Moniuszkistrasse; 5 - Elbląg-Pole Nowomiejskie; 6 - Grochy Stare; 7 - Gronowo; 8 - Grzybnica; 9 - Jasionowa Dolina; 10 - Kowalewko; 11 - Krosno; 12 - Kutowa, Fpl. I; 13 - Kutowa, Fpl. II; 14 - Leśno; 15 - Lubieszewo; 16 - Lubowidz; 17 - Mściszewice; 18 - Nadkole; 19 - Niedanowo; 20 - Nowy Łowicz; 21 - Odry; 22 - Połowite; 23-28 - Pruszcz Gdański, Fpl. 2, 4-7, 10; 29 - Rostołty; 30 - Szpaki; 31 - Weklice; 32 - Węsiory.

Stufe C1 datieren. In Lubowidz mit etwa 300 nicht zerstörten Bestattungen vorwiegend aus der Stufe B2/C1 zählen dagegen zu den Importen nur ein Fragment eines Glasgefäßes und Glasperlen, darunter zwei mit Gesichtdarstellungen (Wołagiewicz 1995). Unbekannt ist, wie viele Gräber in Lubowidz während der Sandgewinnung zerstört wurden; aus diesem Teil stammt ein importierter Spinnrocken aus Bernstein. Die Funde römischer Spinnrocken aus Bernstein sind ausserordentlich selten; sie wurden bisher lediglich in Lubowidz, Czarnówko, Grab R380, Pruszcz Gdański, Fpl. Zuckerfabrik und Kowanówko, Kr. Oborniki, entdeckt (Schuster 2018, 97-101, Abb. 43). Der Spinnrocken von Lubowidz deutet darauf hin, dass sich im zerstörten Teil des Gräberfeldes reich ausgestattete Gräber befunden haben könnten. Allerdings wären in den zahlreichen, noch vor dem Beginn der systematischen Ausgrabungen im Jahre 1939 von H. Agde publizierten Kurzberichten (Wołagiewicz 1995, 11) Funde von Bronze- bzw. Glasgefässen sicher erwähnt worden, wenn diese tatsächlich gefunden worden wären.

Das nächste Beispiel sind die Gräberfelder auf der Elbinger Höhe. Hier stoßen wir auf erhebliche Probleme, da zwei Nekropolen in Elbląg (Elbing) selbst, Elbląg-Pole Nowomiejskie (damals Neustädter Feld) und Elbląg-Moniuszkistrasse (damals Scharnhorststrasse) zerstört sind und ihr Fundmaterial vermischt ist. Die beiden sind nur lückenhaft aus den Ausgrabungen aus dem 19. Jh. und aus der
Zeit vor dem Zweiten Weltkrieg (Natuniewicz 2000, 108-112, dort die ältere Literatur) bekannt. Drei weitere, ebenfalls nur aus einigen Kurzberichten bekannte Nekropolen, sind von der Stadt Elbląg einige Kilometer entfernt.

Entlang der westlichen und südlichen Ränder der Elbinger Höhe befinden sich nach J. OkuliczKozaryn (1992, 139) und A. Cieśliński (2010, 151, 154, Karte 3; 4) einige Dutzend Nekropolen und fast doppelt so viel Siedlungsspuren. Die Blütezeit der Besiedlung datiert hier ab der Stufe B2. Die Nekropole in Weklice, $12 \mathrm{~km}$ von Elbląg entfernt und seit 1984 systematisch untersucht, nimmt hier einen besonderen Platz ein. Etwa 500 Grabinventare sind bereits veröffentlicht (Natuniewicz-Sekuła 2015-2016; Natuniewicz-Sekuła/Okulicz-Kozaryn 2007; 2011). Das mit Ausnahme einer späten Bestattung, von B1b bis C2b belegte Gräberfeld, hat mehrere silberne Trachtbestandteile und Schmucksachen sowie Perlenketten aus bunten Glasperlen geliefert. Zu den bereits publizierten Grabinventaren zählen neun mit sehr reicher Ausstattung, u. a. mit monströsen Fibeln mit Gold- und Silberfolie, römischen Scheibenfibeln, Bronze- und Glasgefässen, terra sigillata, sogar ein glasierter Kantharos wurde gefunden. Grab 150 enthielt einen Amulettsatz, eine Perlenkette, einen verzierten Gürtel und Fibeln mit Pressblechauflagen mit Ansichtsmasken. Die hier beigesetzte, über 60-jährige Dame wird als eine Seherin interpretiert; ob das zutrifft, sei dahingestellt - jedenfalls war ihre soziale Stellung sicher sehr hoch. 
Etwas weiter südlich, in der Iława-Seeplatte, liegt die Nekropole in Połowite (Pollwitten), Kr. Ostróda (Cieśliński 2010, Nr. 124; Eggers 1964), von der 27 Gräber und Streufunde bekannt sind. In sechs Bestattungen wurden zwölf Glasbecher E 189 entdeckt, dazu kommen ein gewellter Bronzeeimer und das Fragment eines zweiten (E 44 und 48). Das Gräberfeld war hauptsächlich in der Stufe Clb belegt. Diese zwei Nekropolen, Weklice und Połowite, sind die reichsten Freidhöfe in der Region.

Das einige Kilometer von Weklice entfernte Gräberfeld von Krosno, Kr. Elbląg, hat dagegen einen unvergleichlich ärmeren Charakter. Nach den Ausgrabungen vom Ende des 19. Jh. und aus der Nachkriegszeit (Andrzejowski/Bursche 1987; Jarzec 2018; Okulicz/Bursche 1987) zu urteilen handelt es sich um eine eine grosse, nehrere hundert Bestattungen zählende Nekropole, jedoch - mit Ausnahme von einigen silbernen Fibeln und Schmucksachen - ohne reichere Beigaben. Die reichste Ausstattung weist Grab 374 auf: vier Fibeln A. II 41, davon zwei aus Silber, und ein Fragment eines silbernen Schlangenkopfarmringes (Jarzed Chowaniec 2017). Im Vergleich zum Reichtum der Nekropole von Weklice macht Krosno den Eindruck eines „armen Verwandten“.

Eine sehr dankbare Grundlage für unsere Vergleichsstudien wäre der Komplex von sechs Gräberfeldern in Pruszcz Gdański an der unteren Weichsel, welche in einer Entfernung von nur zwei Kilometern zu beiden Seiten des Flusses Radunia liegen. Sie sind leider aus verschiedenen Gründen nur teilweise untersucht (Pietrzak 1988; 1997, Abb. 1). Im Falle des vollständig publizierten Gräberfeldes Fpl. 10 (Pietrzak 1997) wurde etwa ein Drittel durch Kiesgrube zerstört; von über 500 geretteten Bestattungen datieren etwa 150 in die römische Kaiserzeit. Nur in einem Grab befanden sich eine Kelle/Sieb-Garnitur E 160, weitere acht können aufgrund einiger Fibeln und langer Halsketten, verzierter Gürtel und dem Fragment eines barbarischen Spinnrockens als reicher gelten. Etwa 40 Grabkomplexe waren sehr arm ausgestattet. Die Nekropole wurde in den Stufen B1-B2/C1 am intensivsten belegt, aus der jüngeren Kaiserzeit stammt nur etwa ein Dutzend Gräber. Das zweite Gräberfeld in Pruszcz Gdański, Fpl. 7, weist ebenfalls mehrere hundert Bestattungen auf, wobei alle Funde aus der Vorkriegszeit verschollen sind. Die Nekropole scheint auch in der jüngeren und späten Kaiserzeit intensiver belegt gewesen zu sein (Pietrzak/Tuszyńska 1988; Tuszyńska 2006). Gerade aus dieser Zeit stammen einige reichen Gräber. Die dritte Nekropole von Pruszcz Gdański, Fpl. 5 - liegt im Bereich eines neuzeitlichen Friedhofs und konnte nur teilweise untersucht werden (Pietrzak/Cymek/Rożnowski 2015). Es wurden 64 Be- stattungen ausgegraben, vorwiegend mit reichen Beigaben, $u$. a. eine Bronzeschüssel mit gekerbtem Rand, ein Glasbecher mit aufgelegten Fäden, eine facettierte Glasschale, alle aus der Stufe D 1, ferner zwei silberne Schildfibeln mit vergoldeten Folien mit Vogeldarstellungen, eine Fibel vom Typ Dybäck/ Independența (Schuster 2017) und mehrere Glas- und Bernsteinketten. Die Belegung dieses Gräberfeldes setzte im Vergleich zu den zwei bereits erwähnten später ein und der Reichtum an Beigaben stammt aus den Stufen C3-D1, als auf den anderen keine Verstorbenen mehr beigesetzt wurden.

Drei weitere Nekropolen von Pruszcz Gdański, "Hartsteinfabrik", "Zuckerfabrik" und „Prauster Dreieck“ (Fpl. 2, 4 und 6) sind nur aus Kurzberichten bekannt; publiziert wurden nur einige Grabkomplexe (La Baume 1926; Schindler 1939; 1941). Aus Grab V vom Gräberfeld "Zuckerfabrik" stammt eine Kelle E 161 (Schindler 1939, 41), von der Nekropole „Prauster Dreieck“ eine Schildfibel aus der Stufe D1 (Schindler 1941).

Das vollständig untersuchte Gräberfeld von Kowalewko, Kr. Oborniki (Skorupka 2001) zählt beinahe 500 Bestattungen, die meistens arm oder beigabenlos waren. 83 Gräber sind durchschnittlich ausgestattet, reiche Gräber gibt es nur acht, u. a. ein mit einer Bronzekanne und eines mit einem Glasbecher E 193. Von anderen Importen sind nur je zwei Scheibenfibeln in vier Gräbern und über 700 Glasperlen aus 60 Grabinventaren zu nennen. Somit kann das Gräberfeld nicht als „reich“ betrachtet werden.

Gehen wir zu den Hügelnekropolen über. Die ältesten Grabhügel in Pommern datieren in die Stufe B1b bzw. den Anfang der Stufe B2. Die Sitte, Hügel zu errichten, dauerte bis zur Stufe C1b, als die Friedhöfe in Pommern verlassen wurden; dagegen datieren die Hügel vom so genannten Rostołty-Typus aus den Gebieten rechts der Weichsel - in Masowien und Podlachien - von C1b bis D1 (Cieśliński 2015, 22 f.). Die Ausstattung der Gräber unter diesen jüngeren Hügeln ist differenziert, worüber noch die Rede sein wird.

Alle 29 Grabhügel der pommerschen Nekropole Gronowo, Kr. Drawsko Pomorskie (Machajewski 2013) wurden untersucht, während die Zone der Flachgräber fast unberührt ist. Die Gräber unter den Hügeln sind reich ausgestattet, u. a. mit insgesamt sechs Bronzegefässen, zwei Spinnrocken einheimischen Typs, Textilienresten vom Typ Virring in drei Gräbern, einem Glasgefäß, 23 Sporen in 12 Gräbern sowie mit Zusammensetzungen des Frauenschmucks, der für den „Barock-Stil“ der Wielbark-Kultur typisch ist - hervorzuheben ist der reichste Grabhügel 22 (Wołagiewicz 1973; 1974). Ein Flachgrab aus dem Jahr 1926 mit nicht ganz 
gesichertem Inventar enthielt neben Sporen zwei Bronzegefässe E 125 und 160 sowie zwei terra sigillata-Teller. Die Hügel über mehreren Gräbern werden als Grabstätten einer Familie interpretiert und ihre Ausstattung weist auf die Entstehung einer privilegierten Schicht hin (Machajewski 2013, 69), welche der Autor der Monographie von Gronowo einige Jahre früher sogar Führungsschicht nannte (Machajewski 2006, 35). Trotz dem Beigabenreichtum sind zehn Gräber in Gronowo entweder beigabenlos oder nur mit einzelnen Gegenständen ausgestattet, wobei die Hügel einen ungestörten Eindruck machen.

Ähnlich reich ausgestattet ist das Hügelgräberfeld von Leśno, Kr. Tuchola. Es wurden hier 13 von insgesamt 15 Grabhügeln ausgegraben. Einige reichen Gräber waren nicht überhügelt, aber mit einem Steinkranz umgeben (Walenta 2009). In zwei der 59 untersuchten Flachgräbern befanden sich Trinkhornbeschläge und verzierte, nicht in Trachtlage deponierte Gürtel; die sonstigen waren arm ausgestattet. $\mathrm{Zu}$ den frühesten Bestattungen zählt hierher Grab 29 mit einer keltischen Silbermünze und zwei Fibeln A. III 52.

Die große Nekropole von Odry, Kr. Chojnice, wie Leśno im Tuchola-Wald gelegen, mit (Stand 2003) 29 Hügeln und 582 Flachgräbern (Grabarczyk 1997, 37-79; 2007; Kmieciński 1968) zeigt ein ganz anderes Bild. Bis auf einige, tatsächlich reiche Bestattungen, jedoch ohne Metallgefässe, wie Hügel 4, Grab 1 und 2 oder auch Grab 127, enthielten die übrigen hinsichtlich Importe nur zwei Fibeln und Halsketten mit einigen Dutzend Glasperlen. Über 300 Gräber der Ausgrabungen von J. Kmieciński sind nie veröffentlicht worden, aber aus den Zusammenstellungen ihrer Inventare in der Arbeit von T. Grabarczyk (1997) geht hervor, dass auch diese gar nicht reich ausgestattet waren. Das Gräberfeld von Węsiory, Kr. Kartuzy (Grabarczyk 2001; Kmieciński/Blombergowal Walenta 1966), mit verhältnismässig reichen - doch ausschliesslich lokalen - Beigaben in den Gräbern unter den Hügeln 6 und 15, kann als relativ arme Nekropole gelten. Ähnlich stellt sich die Lage in Babi Dół-Borcz, Kr. Kartuzy vor, das 15 km von Węsiory entfernt liegt (Mączyńska/Jakubczyk 2017a; 2017b). Die Nekropole wurde vollständig untersucht und umfasst fünf Grabhügel und 114 Flachgräber. Die Gräber, mit wenigen Ausnahmen vom Typ "Wielbark-Barock", sind entweder arm oder beigabenlos; an Importen sind nur zwei Fibeln und kleine Glasperlen zu nennen, an kostbaren Gegenständen zwei goldene Fingerringe aus einem stark gestörten Flachgrab. Es ist jedoch anzumerken, dass mehrere Bestattungen von Babi Dół gründlich gestört waren. Eine arme Ausstattung ist ebenfalls für Grzybnica, Kr. Koszalin charakteristisch, wo Gräber mit drei
Fibeln bzw. mit längeren Perlenketten selten sind (Hahuła/Wołagiewicz 2001).

Sehr interessant ist die Ausstattung von sekundären Gräbern der Wielbark-Kultur in den Hügeln der pommerschen, hallstattzeitlichen Kultur in Mściszewice, Kr. Kartuzy (Amtlicher Bericht 1902, 40-43; 1903, 37 f.; 1907, 27 f.; Janiak 2010; La Baume 1934, 130-133; Skóra 2010, 29-31). Noch im 19. Jh. wurden hier etwa 350 Hügel registriert, von denen nur einige bis heute erhalten blieben. Die sekundären Bestattungen der Wielbark-Kultur enthielten u. a. Gefäße E 44 und 193, Sporen und Silberfibeln, also Beigaben der höheren sozialen Schicht, einige waren allerdings beigabenlos. Die gewellten Eimer E 44-49 konzentrieren sich in Südskandinavien und an der südlichen Ostseeküste (Quast 2009, 120-123, Abb. 7; Schuster 2018, 41, Abb. 11; Tejral 2006, Abb. 25). Nach D. Quast bilden sie den Horizont, der sich mit den Lübsow-Gräbern vergleichen lässt, aber später datiert (B2/C1-C1a), die Gräber mit solchen Metallgefässen sind als Bestattungen lokaler Eliten zu interpretieren. Die Nekropole von Mściszewice ist überhaupt ein sehr interessantes Beispiel, da sich die Gräber der Wielbark-Kultur ausschliesslich in viel älteren Hügeln befanden. Leider ist völlig unbekannt, ob dort auch Flachgräber entdeckt wurden.

In der Studie von K. Skóra (2010, 29-32) sind einige weiteren Beispiele von Wielbark-Bestattungen in den älteren Hügeln angeführt worden, wie in Leśno und Nowy Łowicz. Die Autorin, ähnlich wie S. Brather $(2009,101)$ meint, dass die älteren Grabkonstruktionen absichtlich von den jüngeren Gesellschaften ausgewählt wurden.

Was die jüngere, bereits erwähnte RostołtyHügelgräbergruppe in Masowien und Podlachien anbelangt, hat J. Jaskanis in seiner Monograpbie (Jaskanis 2012, 195-239, dort die ältere Literatur) einen Vergleich dieser Hügel von elf, nur wenige Dutzend Kilometer voneinander entfernten Fundplätzen in Podlachien durchgeführt. Den zentralen Platz nehmen die Nekropolen in Rostołty, Grochy Stare und Szpaki ein, die - obwohl gestört - zugleich am reichsten ausgestattet sind. Ihr Invetar bestand $u$. a. aus Bronze- und Glasgefässen E 161, 189, 192, 209, pressblechverzierten Gegenständen, gläsernen Spielsteinen, Gemmen und zerschnittenem Goldschmuck. Die ältesten sind Hügel in Rostołty, die ab der Stufe B2/C1 angelegt wurden, die Mehrzahl der Hügel der Zentralgruppe datiert in die Stufen C1b-C2. Die peripher gelegenen Hügel, wie Jasionowa Dolina und Kutowa, Fpl. I, datieren jünger und reichen in die Stufe D1 hinein. Sie scheinen viel ärmer ausgestattet gewesen zu sein. Auch die Flachnekropolen in unmittelbarer Nachbarschaft der Hügelnekropolen, wie Cecele (Jaskanis 1996) und Kutowa, Fpl. II, weisen eine durchschnittliche und arme Ausstattung auf. 
J. Jaskanis hält die reichen Rostołty-Hügel für die Grabstätten der Führungsschicht, ohne jedoch eine tiefere Begründung zu liefern.

Zwei Jahre nach dem Erscheinem der Monographie von J. Jaskanis hat A. Cieśliński (2014) die Rostołty-Hügel im breiteren Kontext analysiert. Auch er hat auf die arme Ausstattung einiger davon aufmerksam gemacht (Cieśliński 2014, 82 f.), und sie mit den pommerschen Hügeln der WielbarkKultur verglichen, die Gräber von Verstorbenen mit durchschnittlichen oder gar keinen Beigaben überdecken. Als ein solches Beispiel kann das ungestörte zentrale Grab eines Mädchens(?) mit armer Ausstattung unter Hügel V von Babi Dół-Borcz (Maczyńska/Jakubczyk 2015) angeführt werden. Der Hügel, der größte der fünf vom Gräberfeld, wurde für eine jugendliche Person errichtet, die jedoch eine besondere Stellung in der dortigen Gesellschaft innegehabt haben muss, da für sie mit großem Arbeitsaufwand ein staatliches Grabmonument geschaffen wurde. Sehr arm ausgestattete Gräber unter Hügeln sind auch von Odry (Grabhügel 20) und Węsiory (Grabhügel 8) bekannt (Grabarczyk 1997, 26-28).

Es scheint, dass eine eindeutige Antwort auf die Frage nach den Ursachen von Unterschieden in der Ausstattung in nahen gelegenen oder sogar benachbarten Nekropolen nicht möglich ist. Oft wird die Entfernung von den großen Handelsstraßen hervorgehoben, was den Zugang zu Luxusgütern unmöglich machte (von Carnap-Bornheim 2006, 113; Nagy 2007, 149; Schuster 2014, 56 f.) oder auch der Bestattungsritus, der nicht verlangte, Reichtum zu manifestieren (Brather 2009, 94; Steuer 1982, 233). Im Fall von Gräberfeldern, die nur ein paar Kilometer voneinander liegen, ist es schwierig zu vermuten, dass nur die bestattende Gemeinschaft eines davon den Zugang zu Handelstransaktionen hatte. In dem Fall, den Erwägungen von H. Steuer (1982, 233 f.) folgend, der als Beispiel zwei frühkaiserzeitliche mitteldeutsche Nekropolen anführt, und zwar eine, durchschnittlich ausgestattete von Wahlitz und eine zweite, $40 \mathrm{~km}$ entfernt davon von Kleinzerbst mit mehreren reichen Bestattungen, ist $\mathrm{zu}$ vermuten, dass Kleinzerbst der Friedhof von gehobenen Mitgliedern der Gesellschaft war, während in Wahlitz die abhängigen Bauern beigesetzt wurden. Nach den Berechnungen desselben Verfassers (Steuer 2007, 356-359) war die Bevölkerungsdichte in der römischen Kaiserzeit relativ hoch und die Entfernung zwischen einzelnen Siedlungen betrug von 2 bis $4 \mathrm{~km}$, während die sogenannten Fürstengräber nicht weniger als $20-30 \mathrm{~km}$ voneinander entfernt lagen.

Mit der Zeit lösten sich die Wirtschafts-, vermutlich auch die Herrschaftszentren auf. Dies ist sehr deutlich am Beispiel der Gräber von Lübsow in Pommern zu sehen. Die hier Bestatteten spielten eine führende Rolle in den Stufen B1-B2a in der Region. Später verlor das Zentrum an Bedeutung und entstanden neue, wie Gronowo, Nowy Łowicz (50 und $80 \mathrm{~km}$ von Lübsow entfernt), Odry im Binnenland und Czarnówko an der Ostseeküste (Maczyńska/Rudnicka 2004, 422-425, Abb. 18; Schuster 2010, 260-262, Abb. 108-110). Andere, wie der Komplex von Pruszcz Gdański an der unteren Weichsel, behielten ihre Stellung während der ganzen Kaiserzeit.

Es scheint, dass der Hauptgrund der Differenzierung der Ausstattung einzelner Gräberfelder in der sozialen Struktur zu suchen ist. Die Unterschiede im Grabritus, obwohl möglich, z. B. im Fall armer, aber großer Hügel, waren hier wahrscheinlich nicht erstrangig. Die hier angeführten Beispiele einiger sehr unterschiedlich ausgestatteten Nekropolen bezeugen, dass wir heute das Problem auf unvergleichbar breiterer Basis diskutieren können, obwohl sich eine endgültige Lösung nicht finden lässt.

\section{LITERATUR}

Amtlicher Bericht 1902 - XXII. Amtlicher Bericht über die Verwaltung der naturhistorischen, archäologischen und ethnologischen Sammlungen des Westpreussischen Provinzial-Museums für das Jahr 1901, 1902.

Amtlicher Bericht 1903 - XXIII. Amtlicher Bericht über die Verwaltung der naturhistorischen, archäologischen und ethnologischen Sammlungen des Westpreussischen Provinzial-Museums für das Jahr 1902, 1903.

Amtlicher Bericht 1907 - XXVII. Amtlicher Bericht über die Verwaltung der naturhistorischen, archäologischen und ethnologischen Sammlungen des Westpreussischen Provinzial-Museums für das Jahr 1906, 1907.

Andrzejowski/Bursche 1987 - J. Andrzejowski/A. Bursche: Archeologia biblioteczna. Cmentarzyska kultury wielbarskiej w Krośnie, stanowisko 1 i w Wielbarku, woj. elbląskie. In: A. Pawłowski (red.): Badania archeologiczne w woj. elblaskim w latach 1980-1983. Malbork 1987, 233-277.

Andrzejowski/Żórawska 2002 -J. Andrzejowski/A. Żórawska: Cmentarzysko kultury wielbarskiej na stan. 1 w Nadkolu, woj. mazowieckie. In: J. Andrzejowski/R. Prochowicz/A. Żórawska (red.): Varia Barbarica. Zenoni Woźniak ab amicis dicta. Monumenta Archaeologica Barbarica. Series Gemina 1. Warszawa - Lublin 2002, 29-80.

Becker 2005 - M. Becker: Bemerkungen zu Aussagekraft und Struktur kaiserzeitlicher Grabinventare. In: J. Müller (Hrsg.): Alter und Geschlecht in ur- und frühgeschichtlichen Gesellschaften. Tagung Bamberg 20.-21. Februar 2004. 
Universitätsforschungen zur prähistorischen Archäologie 126. Bonn 2005, 151-155.

Brather 2009 - S. Brather: Tod und Bestattung im frühen Mittelalter. Repräsentation, Vorstellungswelten und Variabilität am Beispiel merowingerzeitlicher Reihengräberfelder. Etnographisch-Archäologische Zeitschrift 50, 2009, 93-115.

von Carnap-Bornheim 2006 - C. von Carnap-Bornheim: Zwischen Anpassung und Widerstand? Überlegungen zu Fürstengräbern der römischen Kaiserzeit im Barbaricum. In: C. von Carnap-Bornheim/D. Krausse/A. Wesse (Hrsg.): Herrschaft - Tod - Bestattung. $\mathrm{Zu}$ den vor- und frühgeschichtlichen Prunkgräbern als archäologisch-historische Quelle. Internationale Fachkonferenz Kiel 16.-19. Oktober 2003. Universitätsforschungen zur prähistorischen Archäologie 139. Bonn 2006, 111-126.

Cieśliński 2010 - A. Cieśliński: Kulturelle Veränderungen und Besiedlungsabläufe im Gebiet der Wielbark-Kultur an Łyna, Pasłęka und oberer Drwęca. Berliner Beiträge zur Vor- und Frühgeschichte. Neue Folge 17. Berlin 2010.

Cieśliński 2014 - A. Ciesliński: Kopce kultury wielbarskiej z Mazowsza i Podlasia a tzw. typ rostołcki - próba nowego spojrzenia na związki cmentarzysk kurhanowych z północnej i wschodniej Polski. Wiadomości Archeologiczne 65, 2014, 45-93.

DOI: https://doi.org/10.36154/wa.65.2014.02

Cieśliński 2015 - A. Ciesliński: Stan i perspektywy badań nad nekropolami kurhanowymi kultury wielbarskiej. In: M. Fudziński/H. Paner (red.): Kontakty ponadregionalne kultury wielbarskiej. Przemiany kulturowe w okresie rzymskim na Pomorzu. Gdańsk 2015, 17-29.

Eggers 1964 - H.-J. Eggers: Das kaiserzeitliche Gräberfeld von Pollwitten, Kreis Mohrungen, Ostpreussen. Jahrbuch des Römisch-Germanischen Zentralmuseums Mainz 11, 1964, 154-175.

DOI: https://doi.org/10.11588/jrgzm.1964.0.35844

Gebühr 1974 - M. Gebühr: Zur Definition älterkaiserzeitlicher Fürstengräber vom Lübsower-Typ. Praehistorische Zeitschrift 49, 1974, 82-136.

Grabarczyk 1997 - T. Grabarczyk: Kultura wielbarska na Pojezierzach Krajeńskim i Kaszubskim. Łódź 1997.

Grabarczyk 2001 - T. Grabarczyk: O Węsiorach w trzydzieści pięć lat później. Pomorania Antiqua 18, 2001, 231-247.

Grabarczyk 2007 - T. Grabarczyk: Wyniki prac wykopaliskowych na cmentarzysku w Odrach, gm. Czersk, woj. pomorskie w latach 1995-2003. Acta Universitatis Lodziensis. Folia Archaeologica 25, 2007, 5-23.

Hahuła/Wołagiewicz 2001 - K. Hahuła/R. Wołągiewicz: Grzybnica. Ein Gräberfeld mit Steinkreisen der WielbarkKultur in Pommern. Monumenta Archaeologica Barbarica 8. Warszawa - Koszalin 2001.

Hedeager 1980 - L. Hedeager: Besiedlung, soziale Struktur und politische Organisation in der älteren und jüngeren römischen Kaiserzeit Ostdänemarks. Praehistorische Zeitschrift 55, 1980, 38-109.

Janiak 2010 - R. Janiak: Czy w Mściszewicach odkryto kolejny pochówek kultury wielbarskiej? In: A. Urbaniak/ R. Prochowicz/I. Jakubczyk/M. Levada/J. Schuster (red.): Terra Barbarica. Studia ofiarowane Magdalenie Mączyńskiej w 65 rocznicę urodzin. Monumenta Archaeologica Barbarica. Series Gemina 2. Łódź - Warszawa 2010, 697-701.
Jarzec 2018 - A. Jarzec: Krosno, stan. 1. Nekropola kultury wielbarskiej z obszaru starożytnego ujścia Wisty. Materiaty $z$ badań w latach 1980-2009. Światowit. Supplement Series B. Barbaricum 12. Warszawa 2018.

Jarzec/Chowaniec 2017 - A. Jarzec/R. Chowaniec: Zestaw srebrnych ozdób z grobu 374 z cmentarzyska kultury wielbarskiej w Krośnie, pow. Pasłęcki. In: J. Andrzejowski/C. von Carnap-Bornheim/A. Cieśliński/ B. Kontny (red.): Orbis Barbarorum. Studia ad archaeologiam Germanorum et Baltorum temporibus Imperii Romani pertinenta Adalberto Nowakowski dedicate. Monumenta Archaeologica Barbarica. Series Gemina 6. Warszawa Schleswig 2017, 373-382.

Jaskanis 1996 - J. Jaskanis: Cecele. Ein Gräberfeld der WielbarkKultur in Ostpolen. Monumenta Archaeologia Barbarica 2. Kraków 1996.

Jaskanis 2012 - J. Jaskanis: Wodzowskie kurhany kultury wielbarskiej na Podlasiu. Białystok 2012.

Kmieciński 1968 - J. Kmieciński (red.): Odry. Cmentarzysko kurhanowe z okresu rzymskiego w powiecie chojnickim. Łódź 1968.

Kmieciński/Blombergowa/Walenta 1966 - J. Kmieciński/ M. Blombergowa/K. Walenta: Cmentarzysko kurhanowe ze starszego okresu rzymskiego w Węsiorach w pow. Kartuskim. Prace i Materiały Muzeum Archeologicznego i Etnograficznego w Łodzi 12, 1966, 37-119.

Kunst 1978 - M. Kunst: Arm und Reich - Jung und Alt. (Untersuchungen zu sozialen Gruppierungen auf dem Gräberfeld vom Hamfelde, Kreis Herzogtum Lauenburg). Offa 35, 1978, 86-109.

La Baume 1926 - W. La Baume: W. La Baume: Ausgrabungen bei Praust, Kreis Danziger Höhe (1925 und 1926). Blätter für deutsche Vorgeschichte 4, 1926, 1-9.

La Baume 1934 - W. La Baume: Urgeschichte der Ostgermanen. Ostlandforschungen 5. Danzig 1934.

Machajewski 2006 - H. Machajewski: Pomorze Środkowe w okresie rzymskim i we wczesnej fazie okresu wędrówek ludów. In: W. Nowakowski/J. Borkowski/ A. Cieśliński/A. Kasprzak (red.): Goci $i$ ich sąsiedzi na Pomorzu. Materialy z konferencji "Goci na Pomorzu Środkowym", Koszalin 28-29 paźdzniernika 2005. Koszalińskie zeszyty muzealne. Seria A. Studia Archaeologica Pomeranica. Tom II. Koszalin 2006, 35-63.

Machajewski 2013 - H. Machajewski: Gronowo. Ein Gräberfeld der Wielbark-Kultur in Westpommern. Monumenta Archaeologica Barbarica 18. Warszawa 2013.

Mączyńska/Jakubczyk 2015 - M. Mączyńska/I. Jakubczyk: „Dziewczynka z zapałkami”? Kurhan V na cmentarzysku kultury wielbarskiej w Babim Dole-Borczu, pow. kartuski, stan. 2. In: B. Kontny (red.): Ubi tribus faucibus fluenta Vistulae fluminis ebibuntur. Jerzy OkuliczKozaryn in memoriam. Światowit. Supplement Series B. Barbaricum 11. Warszawa 2015, 477-488.

Mączyńska/Jakubczyk 2017a-M. Mączyńska/I. Jakubczyk: Cmentarzysko kultury wielbarskiej w Babim Dole-Borczu, pow. Kartuzy, stan. 2. Wstępne podsumowanie wyników badań. In: M. Fudziński/W. Świętosławski/W. Chudziak (red.): Pradoliny pomorskich rzek. Kontakty kulturowe i handlowe społeczeństw w pradziejach i wczesnym średniowieczu. Gdańsk 2017, 214-222.

Mączyńska/Jakubczyk 2017b-M. Mączyńska/I. Jakubczyk: Das Gräberfeld der Wielbark-Kultur von Babi DółBorcz, Kr. Kartuzy, FSt. 2. In: B. V. Eriksen/A. Abegg- 
Wigg/R. Bleile/U. Ickerodt (Hrsg. - eds.): Interaktion ohne Grenzen. Beispiele archäologischer Forschungen am Beginn des 21. Jahrhunderts. Band I - Interaction without borders. Exemplary archaeological research at the beginning of the $21^{\text {st }}$ century. Volume I. Schleswig 2017, 249-256.

Mączyńska/Rudnicka 2004 - M. Mączyńska/D. Rudnicka: Grab mit römischen Importen aus Czarnówko, Kr. Lębork (Pommern). Germania 82, 2004, 397-429.

Nagy 2007 - L. Nagy: Beiträge zur Frage der gesellschaftlichen Stellung der in den römerzeitlichen Hügelgräbern Bestatteten in Transdanubien. Acta Archaeologica Academiae Scientiarum Hungaricae 58, 2007, 145-163. DOI: https://doi.org/10.1556/aarch.58.2007.1.5

Natuniewicz 2000-M. Natuniewicz: „Nowe” znaleziska ze starych wykopalisk. Ocalałe materiały archeologiczne z okresu wpływów rzymskich z okolic Elbląga. In: J. Kolendo/W. Nowakowski (red.): Antiquitates Prussiae. Studia $z$ archeologii dawnych ziem pruskich. Warszawa 2000, 105-206.

Natuniewicz-Sekuła 2015-2016 - M. Natuniewicz-Sekuła: Weklice, stan. 7, pow. elbląski, woj. warmińsko-mazurskie. Badania w latach 2015-2016. Światowit 13-14 (54-55), fascykul A/B, 2015-2016, 235-240.

Natuniewicz-Sekuła/Okulicz-Kozaryn 2011-M. NatuniewiczSekuła/J. Okulicz-Kozaryn: Weklice. A Cemetery of the Wielbark Culture on the Eastern Margin of Vistula Delta (Excavations 1984-2004). Monumenta Archaeologica Barbarica 17. Warszawa 2011.

Okulicz/Bursche 1987 - J. Okulicz/A. Bursche: Badania birytualnego cmentarzyska kultury wielbarskiej w Krośnie na stanowisku $1 \mathrm{w}$ województwie elbląskim. In: A. Pawłowski (red.): Badania archeologiczne w woj. elblaskim w latach 1980-1983. Malbork 1987, 207-231.

Okulicz-Kozaryn 1992 - J. Okulicz-Kozaryn: Centrum kulturowe z pierwszych wieków naszej ery u ujścia Wisły. Barbaricum 2, 1992, 137-155.

Pietrzak 1988 - M. Pietrzak: Cmentarzyska z Pruszcza Gdańskiego w młodszym okresie rzymskim. In: J. Gurba/A. Kokowski (red.): Kultura wielbarska w młodszym okresie rzymskim (materialy z konferencji). Tom I. Lublin 1988, 51-65.

Pietrzak 1997 - M. Pietrzak: Pruszcz Gdański, Fundstelle 10. Ein Gräberfeld der Oksywie- und Wielbark-Kultur in Ostpommern. Monumenta Archaeologica Barbarica 4. Kraków 1997.

Pietrzak/Cymek/Rożnowski 2015 - M. Pietrzak/L. Cymek/ F. Rożnowski: Pruszcz Gdański, stanowisko 5. Cmentarzysko z późnego okresu wpływów rzymskich i wędrówek ludów. Gdańsk 2015.

Pietrzak/Tuszyńska 1988 - M. Pietrzak/M. Tuszyńska: Période romaine tardive (Pruszcz Gdański 7). Inventaria Archaeologica Pologne 60. Warszawa 1988.

Quast 2009 - D. Quast: Frühgeschichtliche Prunkgräberhorizonte. In: M. Egg/D. Quast (Hrsg.): Aufstieg und Untergang. Zwischenbilanz des Forschungsschwertpunktes "Eliten“. Monographien des Römisch-Germanischen Zentralmuseums 82. Mainz 2009, 107-142.

Schindler 1939 - R. Schindler: Neue Gräberfunde aus Praust. Gothiskandza 1, 1939, 38-51.

Schindler 1941 - R. Schindler: Ein Grabfund der Völkerwanderungszeit aus Praust. Gothiskandza 3, 1941, 53-56.
Schuster 2010 - J. Schuster: Lübsow. Älterkaiserzeitliche Fürstengräber im nördlichen Mitteleuropa. Bonner Beiträge zur Vor- und Frühgeschichtlichen Archäologie 12. Bonn 2010.

Schuster 2014 - J. Schuster: Złoty wiek - Czarnówko w okresie wpływów rzymskich i w okresie wędrówek ludów - Golden Age - Czarnówko during the Roman Period and the Migration Period. In: J. Andrzejowski (red. - ed.): Okruch złota w popiele ogniska... Starożytne nekropole w Czarnówku i ich tajemnice - A Fleck of Gold in the Ashes... Mysteries of the Prehistoric Cemeteries in Czarnówko. Monumenta Archaeologica Barbarica. Series Popularis 1. Lębork - Warszawa 2014, 53-89.

Schuster 2017 - J. Schuster: Fibeln vom Typ Dybäck. Independența als Beispiel weitreichender Kontakte zwischen Nord- und Südosteuropa an der Schwelle zur Völkerwanderungszeit. In: Na hranicích impéria. Extra fines imperii. Jaroslavu Tejralovi k 80. narozeninám. Brno 2017, 371-380.

Schuster 2018 - J. Schuster: Czarnówko, Fpl. 5. Acht Prunkgräber - Zeugnisse neuer Eliten im 2. Jh. n. Chr. im Ostseeraum. Monumenta Archaeologica Barbarica 19. Lębork - Warszawa 2018.

Skóra 2010 - K. Skóra: Intruz w kurhanie? O pochówkach wtórnych w obrzędowości pogrzebowej kultur wielbarskiej i przeworskiej. In: K. Skóra/T. Kurasiński (red.): Wymiary inności. Nietypowe zjawiska w obrzędowości pogrzebowej od pradziejów po czasy nowożytne. Acta Archaeologica Lodziensia 56. Łódź 2010, 27-43.

Skóra 2015 - K. Skóra: Struktura społeczna ludności kultury wielbarskiej. Łódź 2015.

Skorupka 2001 - T. Skorupka: Kowalewko 12. Cmentarzysko birytualne ludności kultury wielbarskiej (od połowy I w. n.e. do poczatku III w. n. e.). Archeologiczne badania ratownicze wzdłuż trasy gazociągu tranzytowego 2 . Wielkopolska, cz. 3. Poznań 2001.

Steuer 1982- H. Steuer: Frühgeschichtliche Sozialstrukturen in Mitteleuropa. Eine Analyse der Auswertungsmethoden des archäologischen Quellenmaterials. Abhandlungen der Akademie der Wissenschaften in Göttingen. Philologisch-historische Klasse 128. Göttingen 1982.

Steuer 2007 - H. Steuer: Besiedlungsdichte, Bevölkerungsgrössen und Heeresstärken während der älteren Römischen Kaiserzeit in der Germania magna. In: G. A. Lehmann/R. Wiegels (Hrsg.): Römische Präsenz und Herrschaft im Germanien der augusteischen Zeit. Der Fundplatz von Kalkriese im Kontext neuerer Forschungen und Ausgrabungsbefunde. Abhandlungen der Akademie der Wissenschaften zu Göttingen. Philologisch-historische Klasse. Folge 3, 279. Göttingen 2007, 337-362.

Tejral 2006 - J. Tejral: Die germanische Giessereiwerkstatt in Pasohlávky (Bez. Břeclav). Ein Beitrag zur Frage der Fernhandels- und Kulturbeziehungen nach den Markomannenkriegen. Památky archeologické 97, 2006, 133-170.

Tuszyńska 2006 - M. Tuszyńska: Zapinki zdobione emalią z cmentarzysk w Pruszczu Gdańskim i Ulkowach, pow. Gdański. In: A. Bursche/R. Ciołek (Hrsg.): Corpus der römischen Funde im europäischen Barbaricum. Polen. Supplement - Band 3. Neue Funde römischer Importe aus Polen III. Warszawa 2006, 51-62. 
Walenta 2009 - K. Walenta: Leśno i mikroregion w okresie rzymskim. Chojnice 2009.

Wołagiewicz 1973 - R. Wołągiewicz: Gronowo 1973. Badania na cmentarzysku kurhanowym z okresu wpływów rzymskich. Materiały Zachodnio-Pomorskie 19, 1973, 129-168.

Wołagiewicz 1974 - R. Wołągiewicz: Zagadnienie stylu wczesnorzymskiego w kulturze wielbarskiej. In: F. Lachowicz (red.): Studia Archaeologica Pomeranica.

Manuskript angenommen am 10. 7. 2021

Übersetzt von Jan Schuster
Profesorowi doktorowi Janowi Żakowi w 25-lecie pracy naukowej. Koszalin 1974, 129-152.

Wołagiewicz 1995 - R. Wołągiewicz: Lubowidz. Ein birituelles Gräberfeld der Wielbark-Kultur aus der Zeit vom Ende des 1. Jhs. v. Chr. bis zum Anfang des 3. Jhs. n. Chr. Monumenta Archaeologica Barbarica 1. Kraków 1995. Ziemlińska-Odojowa 1999 - W. Ziemlińska-Odojowa: Niedanowo. Ein Gräberfeld der Przeworsk- und WielbarkKultur in Nordmasowien. Monumenta Archaeologica Barbarica 7. Kraków 1999.

prof. dr hab. Magdalena Mączyńska

Bobrzyńskiego 43/61

PL - 38-340 Kraków

magdalena.babidol@gmail.com 